\title{
The role of FGF-2 in smoke-induced emphysema and the therapeutic potential of recombinant FGF-2 in patients with COPD
}

You-Sun Kim', Goohyeon Hong ${ }^{2}$, Doh Hyung Kim², Young Min Kim $\mathbb{D}^{2}$, Yoon-Keun Kim³ ${ }^{3}$ Yeon-Mok Oh ${ }^{1}$ and Young-Koo Jee ${ }^{2}$

\begin{abstract}
Although the positive effects of recombinant fibroblast growth factor-2 (rFGF-2) in chronic obstructive pulmonary disease (COPD) have been implicated in previous studies, knowledge of its role in COPD remains limited. The mechanism of FGF2 in a COPD mouse model and the therapeutic potential of rFGF-2 were investigated in COPD. The mechanism and protective effects of $\mathrm{FFGF}-2$ were evaluated in cigarette smoke-exposed or elastase-induced COPD animal models. Inflammation was assessed in alveolar cells and lung tissues from mice. FGF-2 was decreased in the lungs of cigarette smoke-exposed mice. Intranasal use of rFGF-2 significantly reduced macrophage-dominant inflammation and alveolar destruction in the lungs. In the elastase-induced emphysema model, rFGF-2 improved regeneration of the lungs. In humans, plasma FGF-2 was decreased significantly in COPD compared with normal subjects ( 10 subjects, $P=0.037$ ). The safety and efficacy of inhaled rFGF-2 use was examined in COPD patients, along with changes in respiratory symptoms and pulmonary function. A 2-week treatment with inhaled rFGF-2 in COPD $(n=6)$ resulted in significantly improved respiratory symptoms compared with baseline levels $(P<0.05)$; however, the results were not significant compared with the placebo. The pulmonary function test results of COPD improved numerically compared with those in the placebo, but the difference was not statistically significant. No serious adverse events occurred during treatment with inhaled rFGF-2. The loss of FGF-2 production is an important mechanism in the development of COPD. Inhaling rFGF-2 may be a new therapeutic option for patients with COPD because rFGF-2 decreases inflammation in lungs exposed to cigarette smoke.
\end{abstract}

\section{Introduction}

Chronic obstructive pulmonary disease (COPD) is a disease entity that is physiologically characterized by chronic irreversible airflow limitation causing progressive decline of lung function and intermittent episodes of

Correspondence: Yeon-Mok Oh (yeonmok.oh@gmail.com) or

Young-Koo Jee (ykjee@dankook.ac.kr)

${ }^{1}$ Department of Pulmonary and Critical Care Medicine, Asan Medical Center,

University of Ulsan College of Medicine, Seoul, Republic of Korea

${ }^{2}$ Department of Internal Medicine, Dankook University College of Medicine,

Cheonan, Republic of Korea

Full list of author information is available at the end of the article.

These authors contributed equally: You-Sun Kim, Goohyeon Hong increased severity of the disease, known as acute exacerbations. The pathophysiological characteristics of COPD comprise several components, such as mucus hypersecretion, oxidative stress, and chronic inflammation of the airways and lungs ${ }^{1}$. Although bronchodilators, such as long-acting $\beta 2$-agonists (LABAs) and long-acting muscarinic antagonists (LAMAs), with or without inhaled corticosteroids (ICSs), are recommended as first-line treatments for symptomatic patients, there is no available treatment option to reverse changes in pulmonary structures and declining lung function. In addition, long-

\section{(-) The Author(s) 2018}

(c) (i) Open Access This article is licensed under a Creative Commons Attribution 4.0 International License, which permits use, sharing, adaptation, distribution and reproduction Ca in any medium or format, as long as you give appropriate credit to the original author(s) and the source, provide a link to the Creative Commons license, and indicate if changes were made. The images or other third party material in this article are included in the article's Creative Commons license, unless indicated otherwise in a credit line to the material. If material is not included in the article's Creative Commons license and your intended use is not permitted by statutory regulation or exceeds the permitted use, you will need to obtain permission directly from the copyright holder. To view a copy of this license, visit http://creativecommons.org/licenses/by/4.0/. 
term use of corticosteroids can cause complications in patients with advanced stage $\mathrm{COPD}^{2,3}$.

The exact mechanism that leads to the presence of pulmonary emphysema, an important part of COPD, is not fully understood. It has been shown that emphysema might be the result of interactions between environmental triggers (such as cigarette smoking) in patients with genetic predisposition. Smoking is known as the main cause of the development of emphysema ${ }^{4}$. Studies have shown that smoking causes an induction of several epigenetic changes in the methylation of DNA, which has been found to occur in emphysema ${ }^{5}$. In the case of emphysema, oxidative stress mainly caused by cigarette smoking results in the development of cellular injury and apoptosis $^{6}$. A common mediator in emphysema is transforming growth factor- $\beta$ (TGF- $\beta$ ). Although TGF is known as a major regulator of fibrosis, it has recently been found to play a significant role in the development of emphysema ${ }^{7}$. FGF signaling regulates TGF- $\beta$-dependent smooth muscle cell phenotypes ${ }^{8}$. In other words, FGF-2 is a downstream mediator of TGF- $\beta$ and is important in wound healing9?.

Fibroblast growth factors (FGFs) are members of the heparin-binding growth factor family that affect the growth and differentiation of various cell types ${ }^{10}$. They are often involved in morphogenesis, wound repair, inflammation, and angiogenesis and function as potent chemotactic and mitogenic factors for cells originating from the mesoderm, ectoderm and endoderm ${ }^{11-14}$. In addition, FGF-2 has attracted increasing attention as an important factor involved in airway remodeling by increasing the deposition of proteoglycans and its eventual association with bronchial hyperresponsiveness in asthmatic airways $^{12-15}$.

Previous studies have suggested that there are beneficial effects of recombinant FGF-2 (rFGF-2) in treating COPD in animal emphysema models ${ }^{16-19}$. In addition, rFGF-2 has been reported to be an inhibitor of inflammatory cells and cytokines related to asthma and has been shown to reduce airway remodeling in an asthma mouse model $^{20}$. In a clinical trial, inhaled rFGF-2 was safely used in patients with asthma, whose daytime and nocturnal symptoms as well as quality of life were improved without any serious adverse events (AEs) ${ }^{21}$. Regardless of these positive results of $\mathrm{rFGF}-2$ for treating animal emphysema models, it is reasonable to assume that rFGF-2 would be effective in patients with COPD based on the similarity between the pathogeneses of COPD and asthma. Furthermore, the development of fundamental medicine to prevent airway hyperresponsiveness and airway remodeling in patients with COPD has been considered a priority.

In this study, the mechanisms of FGF-2 in the development of COPD (especially emphysema) and the therapeutic potential of FGF2 were explored in a cigarette smoke-exposed C57BL/6 J emphysema mouse model. Additionally, a prospective placebo-controlled pilot study was conducted separately to define the potential role of rFGF-2 in improving or stabilizing symptoms and lung function in patients with COPD.

\section{Materials and Methods}

\section{Animal experiments}

\section{Animal model}

C57BL/6 J mice were purchased from Orientbio (Seongnam, Korea). The mice were bred in specific pathogen-free facilities at Asan Medical Center. Female C57BL/6 J mice (7 weeks of age) were exposed to commercial cigarette smoke based on the protocol used in our previous report ${ }^{22}$.

Briefly, mice were exposed to smoke for 1 day, 4 days, and 6 months ( 5 days/week) in an inhalation box (50 $\times$ $40 \times 30 \mathrm{~cm})$ connected to a pump to assess changes in FGF-2 levels. To evaluate the effect of short-term smoke exposure on the mice, mice were exposed to smoke for 4 days, and $0.1,10$, or $1,000 \mathrm{pg}$ of rFGF- 2 was intranasally administered to each mouse immediately after smoke exposure on each day to define the effects of rFGF-2 on smoke exposure. After completing the 4-day smoke exposure with or without rFGF-2, the mice were killed to collect their lung tissues on day 5 .

For the elastase-induced mouse model, $0.4 \mathrm{U}$ of porcine elastase (Sigma-Aldrich, St. Louis, MO, USA) was intratracheally injected into C57BL/6 mice on day 0. Then, rFGF-2 $(10 \mathrm{pg})$ was administered intranasally once daily from days 7-13, and PD173074 was intraperitoneally injected once per day during the same period. The mice were killed to collect their lung tissues on day 14 .

All mouse experiments were approved by the Institutional Animal Care and Use Committee of Asan Medical Center (South Korea).

\section{Bronchoalveolar lavage cellularity}

Cellularity in Bronchoalveolar lavage (BAL) fluid was analyzed as previously described ${ }^{23}$. In brief, BAL fluid was obtained from both lungs using PBS, and the cells were collected after centrifugation. The total number of cells in the BAL fluid was counted after trypan-blue staining, and the cell pellet was diluted with $500 \mu \mathrm{L}$ of PBS for counting. The BAL differential cell count was determined by DiffQuick staining in 300 inflammatory cells counted. Inflammatory cells were classified as macrophages, lymphocytes, neutrophils or eosinophils.

\section{Histology and quantification of emphysema}

The lungs were perfused with PBS and inflated by intratracheal infusion of $0.5 \%$ low-melting agar in $25 \mathrm{~cm}$ $\mathrm{H}_{2} \mathrm{O}$, fixed in $4 \%$ paraformaldehyde and embedded in paraffin. Lung sections of $4-\mu \mathrm{m}$ thickness were stained 
with hematoxylin and eosin (H\&E). The mean linear intercept (MLI) was determined separately by 2 investigators in a blinded fashion ${ }^{22}$.

\section{Caspase-3/7 activity and western blotting}

A $10-\mu g$ portion of lung protein homogenized in RIPA buffer (Cell Signaling Technology, Danvers, MA, USA) was measured with the Apo-one homogeneous caspase-3/ 7 assay kit (Promega, Madison, WI, USA) according to the manufacturer's protocol. After $5 \mathrm{~h}$, sample fluorescence was measured using a fluorometer. Western blotting using anti-caspase-3 polyclonal antibody (Enzo Life Science, New York, NY, USA) was performed to quantify pro- and active caspase- 3 in lung tissues.

\section{Elastase activity}

Lung tissues were homogenized with protein lysis buffer (Cell Signaling Technology) without protease inhibitors and subjected to the EnzCheck Elastase Assay Kit (Molecular Probes, Sunnyvale, CA, USA) according to the manufacturer's protocol.

\section{FGF-2}

FGF-2 was measured in the plasma of normal controls and patients with COPD (10 subjects/group). The lungs of mice were homogenized in RIPA buffer, and the quantity of protein was assessed with the Bradford assay. FGF-2 levels were measured using an enzyme-linked immunosorbent assay (ELISA) (R\&D Systems, Minneapolis, MN, USA) according to the manufacturer's protocol.

\section{Clinical trial: a pilot study}

This single-center, double-blind, randomized, placebocontrolled pilot trial was conducted at Dankook University Hospital between October 2009 and September 2010. The study protocol was approved by the Korean Food and Drug Administration and the Institutional Review Board at Dankook University Hospital (DKUH 0909-066). Written informed consent was obtained from all patients before they participated in the study. Dong-A Pharm. Co. Ltd. (Seoul, South Korea) provided the rFGF2. The dosage used in this study was determined based on a previous clinical trial performed in patients with asthma $^{21}$.

Randomization was performed using a random number table on day 1 (screening visit). Eligible patients with COPD were randomly allocated to $4.5 \mathrm{ng}$ /day $\mathrm{rFGF}-2$ or a placebo. In the treatment group, $1.5 \mathrm{ng}$ of rFGF-2 was administered over $10 \mathrm{~min}, 3$ times a day for 2 weeks using a nebulizer kit (PARI BOY ${ }^{\circledR}$ type 085; PARI GmbH, Starnberg, Germany), and normal saline was administered to the placebo group using the same method for the same duration. The biological half-life of FGF is $<50 \mathrm{~min}^{24}$, which is too short for a single administration by inhalation to be sufficient because wound healing takes longer than 1 day $^{25}$. Therefore, administration 3 times a day was recommended for this clinical pilot trial. Concomitant use of ICSs, LABAs/LAMAs, mucus-active agents, and antitussives was permitted during the study, but use of systemic corticosteroids was prohibited to avoid any potential confounding effects on the clinical endpoints.

Twelve patients with COPD were randomly assigned to a 2-week treatment period $(n=6)$ or placebo $(n=6)$, followed by a 4-week nontreatment follow-up period. Patients were first seen at a screening visit and afterwards at 7,15 , and 28 days after completion of the trial. At screening and at each visit, the following results were recorded: physical examination including vital signs, pulmonary function tests (PFTs), chest radiography, and electrocardiogram (ECG); and blood chemistry, urinalysis, and dyspnea scale including the American Thoracic Society (ATS) dyspnea scale and the modified Borg scale. Patients had to meet all the criteria included in Table S2 in the Supplementary Appendix.

Patients were not eligible for the trial if they met 1 or more of the exclusion criteria listed in Table S3. The clinical characteristics are displayed in Table S3. The primary endpoint was confirmation of the safety of inhaled rFGF-2. Safety was assessed by reporting AEs, vital signs, chest radiography, ECG, and laboratory test results, including complete blood count, blood chemistry, and urinalysis. The secondary endpoints were the clinical effects of rFGF-2 from baseline to 4 weeks of treatment. The clinical effects were assessed by analyzing PFT results, such as changes in forced expiratory volume in $1 \mathrm{~s}$ (FEV1), forced vital capacity (FVC), the Global Chest Symptoms Questionnaire (GCSQ) ${ }^{26}$, as well as dyspnea scales including the ATS dyspnea scale and the modified Borg scale.

Data are presented as the mean and standard deviation for continuous variables and as a number (percentage) for categorical variables. The Mann-Whitney $U$ test was used to compare plasma FGF-2 levels between the COPD and control groups. Two-way analysis of variance (ANOVA) was performed for BAL cellularity. In the human clinical study, differences between the study and control groups were tested using the Wilcoxon matched pair signed-rank test. A $P$ value of $<0.05$ was considered statistically significant. The data were analyzed using PASW statistics ver. 18 (SPSS Inc., Chicago, IL, USA).

\section{Results}

FGF-2 production in the lungs is dependent on exposure time to cigarette smoke

To evaluate the effects of exposure to cigarette smoke on FGF-2 production in the lungs, the amounts of FGF-2 in lung lysates were compared using ELISA in C57BL/6 

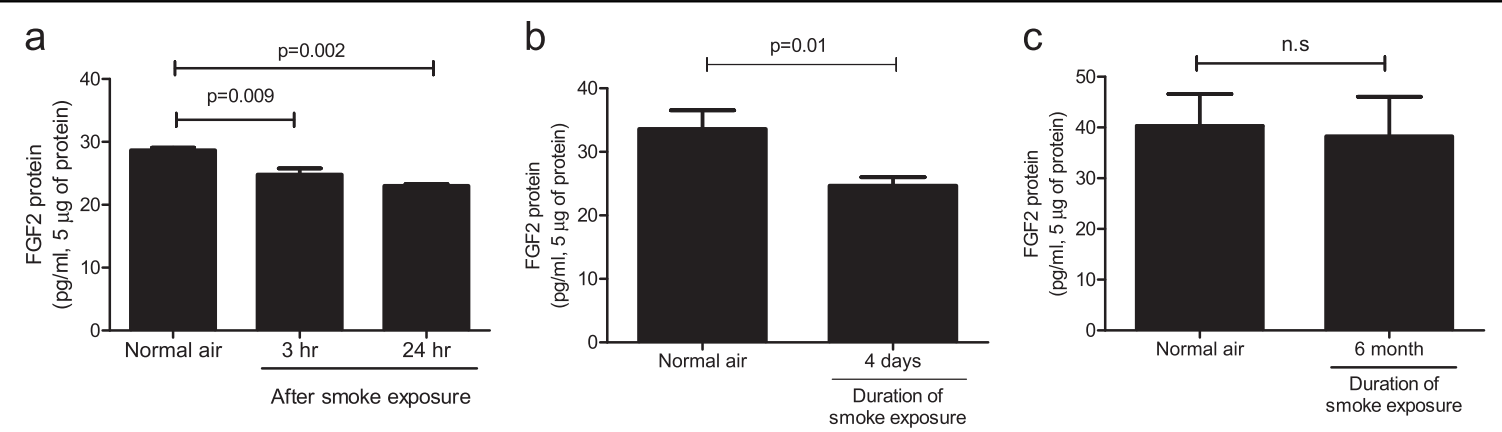

Fig. 1 Fibroblast growth factor-2 (FGF-2) levels in lung lysates decreased in response to short-term smoke exposure. a-c FGF-2 levels were measured in $5 \mathrm{\mu g}$ of lung lysate using an enzyme-linked immunosorbent assay. a Mice were exposed to cigarette smoke once, and lung tissues were obtained 3 and $24 \mathrm{~h}$ after exposure ( $n=6$ per group). b. c Mice were exposed to cigarette smoke for 4 days or 6 months, and lung tissues were collected $18 \mathrm{~h}$ after the last exposure (b $n=12$ per group, $\mathbf{c} n=5$ per group). $P$-values were determined by the Mann-Whitney test

mice. Compared with mice exposed to normal air, FGF-2 production in lung tissues was decreased 3 and $24 \mathrm{~h}$ after smoke exposure in mice exposed to smoke once (Fig. 1a). A decrease in FGF-2 levels was also observed in mice exposed to smoke for 4 days (Fig. 1b). However, the level of FGF-2 produced in mice exposed to smoke for 6 months was not significantly different from that in mice exposed to normal air (Fig. 1c). Therefore, FGF-2 production was decreased in mouse lungs only after shortterm exposure to cigarette smoke.

\section{Emphysema induced by short-term exposure to cigarette smoke in mice}

To determine the effects of a decreased FGF-2 level on the lungs after short-term exposure to cigarette smoke (presented in Fig. 1), BAL fluid and lung tissue were collected on day 5 from C57BL/6 mice exposed to cigarette smoke for 4 days, and inflammation and the emphysema index representing the COPD patient's phenotype were determined. Recruitment of inflammatory cells was increased in smoke-exposed mice; macrophages were the main inflammatory cell type that was increased in the BAL fluid of the smoke-exposed mice (Fig. 2a). The MLI was measured using H\&E-stained lung tissue to determine the index of alveolar destruction, which was significantly increased in smoke-exposed mice compared with that in normal air-exposed mice (Fig. 2b). Lung resident cell apoptosis and protease activity developed in the destroyed alveoli. Apoptosis based on caspase-3/ 7 signaling in lung tissue was increased more in mice exposed to cigarette smoke than in those exposed to normal air (Fig. 2c), but alveolar destruction observed in smoke-exposed mice was not associated with an induction of elastase activity (Fig. 2d). Overall, mice that were exposed to short-term smoke showed phenotypes similar to those of patients with COPD, such as macrophagedominant inflammation and alveolar destruction.
Protective effect of rFGF-2 on the development of emphysema induced by exposure to short-term cigarette smoke in mice

To confirm the protective effect of rFGF-2, 3 doses (0.1, 10 , and $1000 \mathrm{pg}$ ) of the rFGF-2 protein were intranasally administered immediately after the mice were exposed to smoke for 4 days. The number of inflammatory cells recruited was decreased more in mice administered 10 or $1000 \mathrm{pg}$ of rFGF-2 than in those administered 0 or $0.1 \mathrm{pg}$ of rFGF-2 after smoke exposure (Fig. 3a). In particular, the numbers of macrophages and lymphocytes were decreased in BAL fluid obtained from the 10 and $1000 \mathrm{pg}$ rFGF-2-administered mice. Alveolar destruction was also decreased in mice administered 10 and $1000 \mathrm{pg}$ of rFGF-2 (Fig. 3b, c). These results suggest that the loss of FGF-2 production is an important initial mechanism of developing COPD in response to cigarette smoke, and this phenomenon was protected by and compensated for using rFGF-2.

\section{Effect of rFGF-2 on apoptosis signaling in cigarette smoke- exposed mice}

Resident lung cell apoptosis developed in the destroyed alveoli of mice exposed to smoke over the short term (Fig. 2). Resident lung cell apoptosis was dependent on caspase-3 signaling in mice exposed to smoke for 4 days. Caspase-3/7 activity was significantly decreased immediately after smoke exposure in mice intranasally administered $10 \mathrm{pg}$ of rFGF-2 each day than in those exposed to smoke alone (Fig. 4a). Pro- and active caspase-3 protein expression was also decreased in the group receiving rFGF-2 (Fig. 4b).

\section{Regenerative effect of $\mathrm{rFGF}-2$ on the development of emphysema in the elastase-induced mouse model}

To evaluate the regenerative effects of FGF-2, rFGF-2 was intranasally administered to mice with emphysema, which was induced by injecting porcine elastase for 


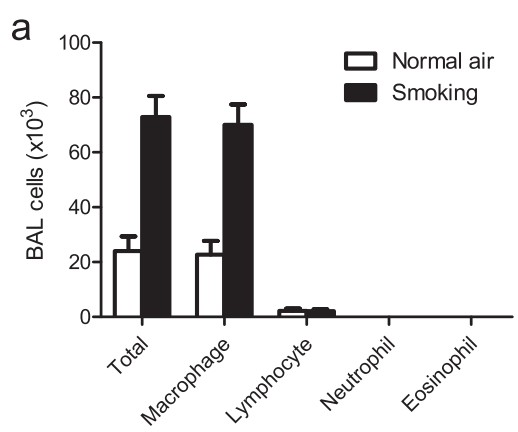

b
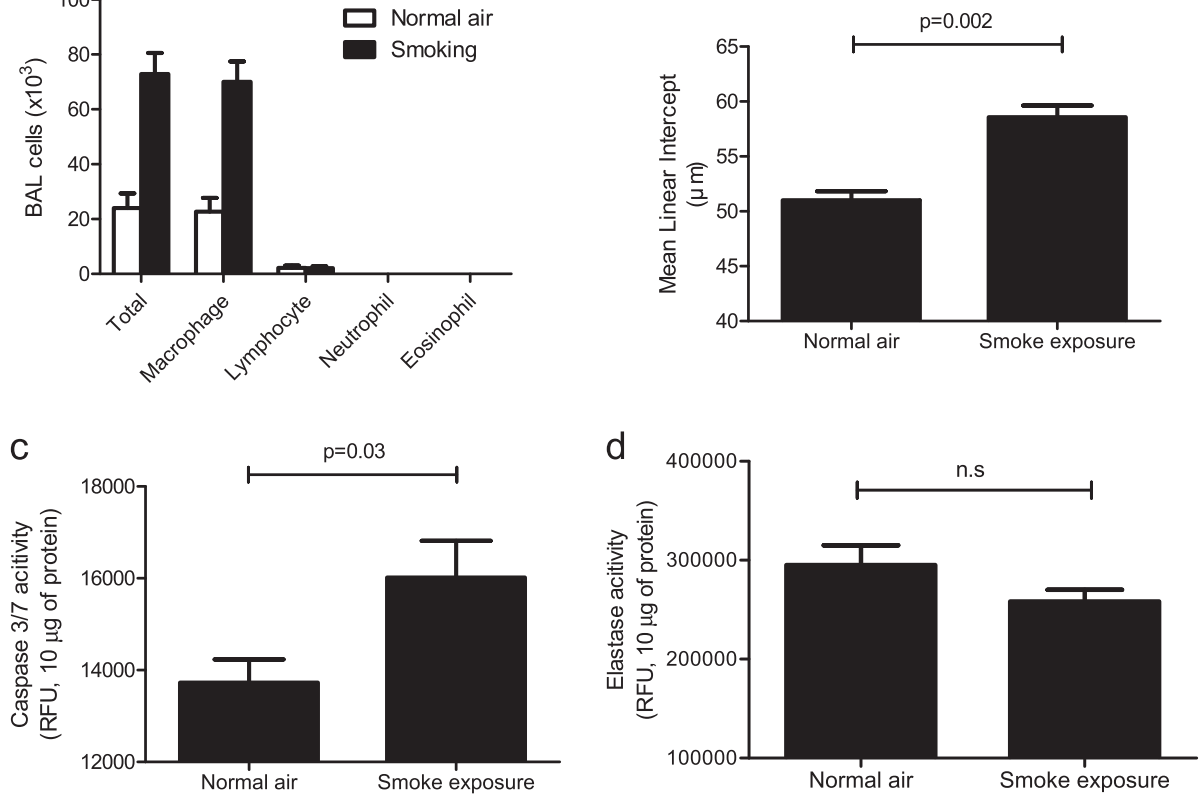

Fig. 2 Characterization of short-term exposure to cigarette smoke. a-d The mice were exposed to cigarette smoke for 4 days using an inhalation box. a Cellularity in bronchoalveolar lavage (BAL) fluid. $P<0.0001$ using two-way analysis of variance. b Mean linear intercept (MLI) $(n=6$ per group). c Caspase-3/7 and elastase activities were measured in $10 \mu \mathrm{g}$ of lung lysate ( $n=11$ per group). $P$-values were determined by the Mann-Whitney test $(\mathbf{b}-\mathbf{d})$
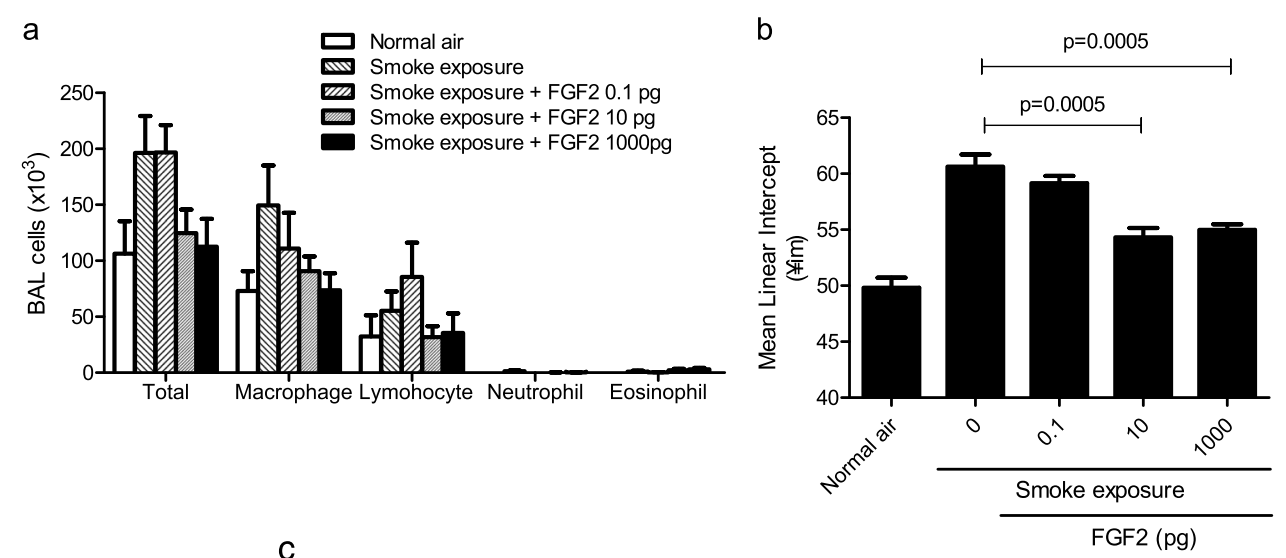

C

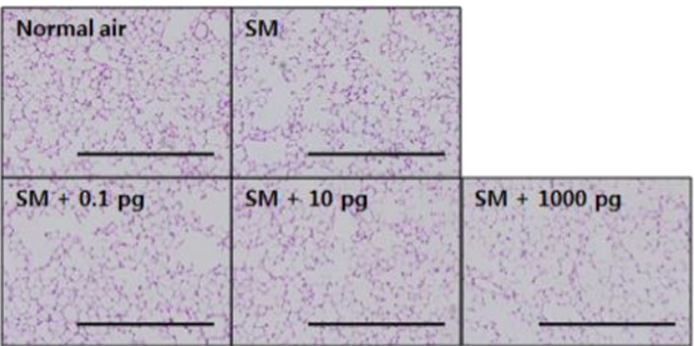

Fig. 3 Protective effects of FGF-2 on early emphysema in response to short-term exposure to cigarette smoke. a-c The mice were exposed to cigarette smoke for 4 days, and $0.1,10$, and $1000 \mathrm{pg}$ of FGF-2 was administered intranasally within $1 \mathrm{~h}$ after smoke exposure. Mice were killed $18 \mathrm{~h}$ after the final smoke exposure. a Cellularity in BAL fluid. $P$-values were determined by two-way analysis of variance. b MLI ( $n=6-12$ per group). $P$ values were determined by the Mann-Whitney test. $\mathbf{c}$ Lung histology with hematoxylin and eosin (H\&E) staining (scale bar $=1 \mathrm{~mm})$ 


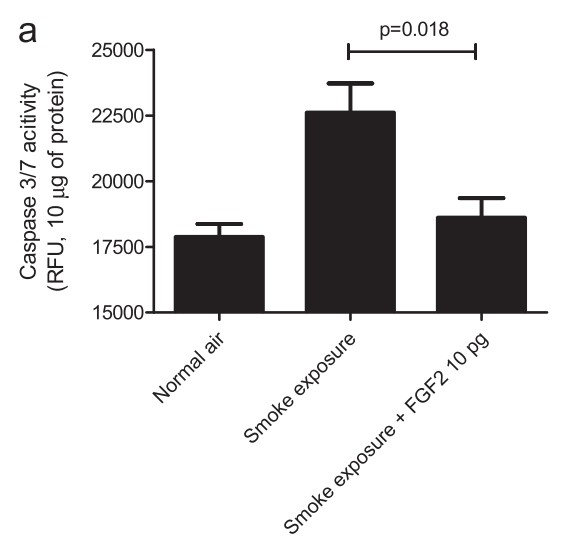

b

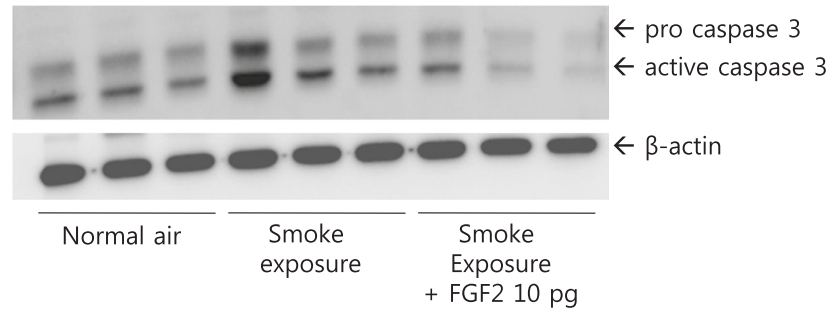

Fig. 4 Lung resident cell apoptosis in response to smoke was protected against by FGF-2 and dependent on caspase-3/7 signaling. $\mathbf{a}, \mathbf{b}$ The mice were exposed to cigarette smoke for 4 days, and $10 \mathrm{pg}$ of FGF-2 was intranasally administered within $1 \mathrm{~h}$ after smoke exposure. Mice were killed $18 \mathrm{~h}$ after the final smoke exposure. a Caspase-3/7 activity using $10 \mu \mathrm{g}$ of lung lysate ( $n=9$ per group). $P$-values were determined by the Mann-Whitney test. $\mathbf{b}$ Western blotting using antibodies against caspase-3 and $\beta$-actin
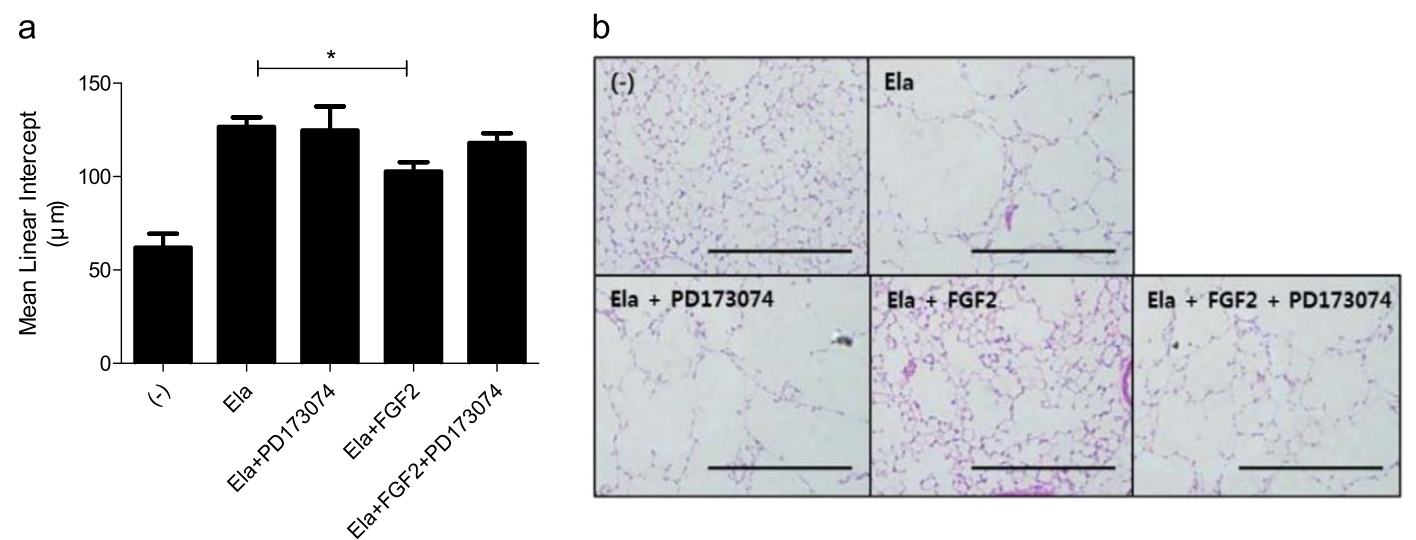

Fig. 5 Regenerative effects of FGF-2 on the elastase-induced emphysema model. The mice were intratracheally injected with $0.4 \mathrm{U}$ of porcine elastase on day 0 . Aliquots (10 pg) of FGF-2 and PD173074 were intranasally and intraperitoneally administered on days 7-13. The mice were killed on day 14. a MLI ( $n=4-6$ per group). $P$-values were determined by the Mann-Whitney test; ${ }^{*} p<0.05$. $\mathbf{b}$ Lung histology with H\&E staining (scale bar $=1$ $\mathrm{mm})$

7 days. The rFGF-2 protein $(10 \mathrm{pg})$ was administered once daily for 4 days after completing the elastase injection schedule. The lungs were effectively regenerated in rFGF-2-treated mice compared with nontreated mice (Fig. 5a, b). To confirm the direct association between emphysematous lung regeneration and FGF-2, we used PD173074 as an FGF receptor $1 / 3$ inhibitor. The regenerative effect shown in elastase-induced emphysematous mice receiving rFGF-2 was not observed when PD173074 was given intraperitoneally (Fig. 5a, b), suggesting a direct association between FGF-2-mediated signaling and the regeneration of emphysematous lungs.

\section{Blood FGF-2 levels in patients with COPD}

To determine whether the blood FGF-2 level is correlated with COPD, FGF-2 levels were measured in plasma from patients with COPD and normal controls using ELISA in 10 subjects each from the study groups. Plasma was obtained from individuals with similar ages and histories (Table S1). The mean plasma FGF-2 level was 102.9 $\mathrm{pg} / \mathrm{mL}$ in patients with COPD and $125.5 \mathrm{pg} / \mathrm{mL}$ in the normal controls $(P=0.037)$ (Fig. 6).

\section{Safety of inhaling FGF-2 in patients with COPD}

The flowchart for the clinical pilot trial is shown in Fig. 7. No treatment-associated severe AEs requiring treatment discontinuation were observed in the rFGF-2 group. No abnormal findings were detected on physical examination in the treatment group during the study period. Petechiae were found in 1 patient in the control group on day 2 of rFGF-2 treatment, but the lesions disappeared without any medication. No abnormal 

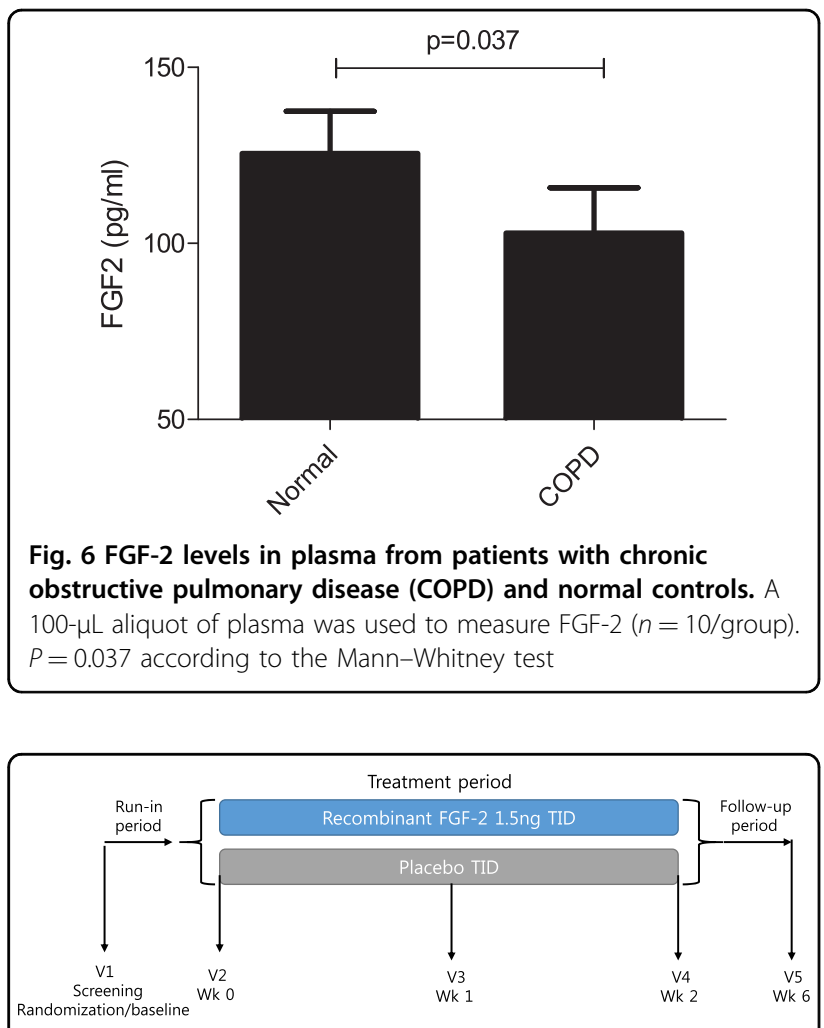

Fig. 7 Flowchart of the clinical pilot trial. V visit; Wk week

findings were observed on chest radiographs, ECGs or laboratory tests, including a complete blood count, blood chemistry, and urinalysis, during the study period.

The most commonly reported AEs were cough and sore throat. The incidences of AEs are summarized in Table S4. The AEs improved spontaneously during treatment with inhaled rFGF-2. Therefore, AEs were not causally related to inhaled rFGF-2 treatment.

\section{Clinical effects of inhaling FGF-2 in patients with COPD}

All patients enrolled in the clinical trial were current smokers. The demographic characteristics of patients are summarized in Table 1. PFTs including FEV1 and FVC tended to improve during the rFGF-2 treatment period, but they were not significantly different between the rFGF-2 and control groups $P>0.05$. Changes in the PFTs are summarized in Table 2 and Fig. 8.

According to the GCSQ results, the grade of dyspnea, chest discomfort, and daytime activities improved more after treatment than before treatment in the rFGF-2 group $(P<0.05)$. The ATS dyspnea scale score improved significantly 4 weeks after treatment compared with the first day of treatment $(P=0.038)$. The modified Borg scale score improved significantly 4 weeks after treatment $(2.5$ $\pm 1.4)$ compared with the first day of treatment $(4.8 \pm 2.0)$ and day 7 of treatment $(4.0 \pm 1.1)(P=0.042$ and $P=$
Table 1 Demographic characteristics of the subjects in the clinical trial

\begin{tabular}{|c|c|c|c|}
\hline & $\begin{array}{l}\text { Control group ( } n \\
=6)\end{array}$ & $\begin{array}{l}\text { FGF-2 group ( } n \\
=6)\end{array}$ & $p$-value ${ }^{a}$ \\
\hline Age (year) & $66.7 \pm 11.7$ & $63.0 \pm 10.5$ & 0.39 \\
\hline Male (\%) & $6(100)$ & $5(83)$ & 0.30 \\
\hline $\mathrm{BMI}\left(\mathrm{kg} / \mathrm{m}^{2}\right)$ & $21.8 \pm 3.3$ & $23.3 \pm 3.6$ & 0.39 \\
\hline $\begin{array}{l}\text { Smoking pack- } \\
\text { years }\end{array}$ & $45.0 \pm 10.9$ & $32.0 \pm 17.3$ & 0.24 \\
\hline \multicolumn{4}{|c|}{ Pulmonary function tests } \\
\hline $\mathrm{FEV}_{1}(\%)$ & $41.6 \pm 9.19$ & $49.7 \pm 7.4$ & 0.24 \\
\hline FVC (\%) & $63.6 \pm 10.5$ & $74.2 \pm 15.2$ & 0.13 \\
\hline
\end{tabular}

FGF-2 fibroblast growth factor-2, BMI body mass index, $F E V_{1}$ forced expiratory volume in $1 \mathrm{~s}, F V C$ forced vital capacity

${ }^{\text {a }}$ Statistical differences were tested by Pearson's chi-square test

0.041 , respectively). Chest discomfort improved significantly on day 15 of treatment $(0.3 \pm 0.5)$ compared with the first day of treatment $(1.7 \pm 1.0)(P=0.038)$. Daytime activities significantly improved more during the 4 weeks after treatment $(12.8 \pm 8.6)$ than during the first day of treatment $(21.8 \pm 9.7)$ and day 15 of treatment (28.3 \pm 14.8$)(P=0.042$ and $P=0.043$, respectively). In particular, the effects of rFGF-2 were maintained for up to 4 weeks after treatment. However, no significant differences were observed between the rFGF- 2 and control groups for GCSQ, grade of dyspnea, chest discomfort, or daytime activities $(P>0.05)$. The dyspnea scales, including the ATS dyspnea scale and the modified Borg scale, were not significantly different between the rFGF- 2 and control groups. These results are summarized in Tables 3 and 4 .

\section{Discussion}

This study revealed several important findings. First, plasma FGF-2 levels were decreased more in patients with COPD than in normal subjects. Second, FGF-2 levels were decreased in short-term smoke-exposed mice. Finally, FGF-2 significantly protected against the development of emphysema induced by short-term exposure to cigarette smoke in mice.

A significant inverse correlation was observed between short-term exposure to cigarette smoke and FGF-2 production, indicating that the degree of pulmonary FGF-2 expression is related to the magnitude of lung exposure to smoke during the early phase. In this study, we tested very short-term exposure to smoke in animal experiments. Biological changes induced by smoke mainly develop during the early phase; therefore, biological changes are not clearly observed in the late phase. The mechanisms involved in this phenomenon are poorly understood. In fact, FGF-2 levels changed upon short-term exposure to 
Table 2 Pulmonary function test $\left(F E V_{1} / \Delta F E V_{1}\right.$ and $\left.F V C / \triangle F V C\right)$ of each group during and after FGF-2 treatment in the clinical trial

\begin{tabular}{|c|c|c|c|c|c|c|c|c|}
\hline & \multicolumn{2}{|l|}{$\mathrm{FEV}_{1}(\%)$} & \multicolumn{2}{|l|}{$\Delta \mathrm{FEV}_{1}(\%)$} & \multicolumn{2}{|l|}{ FVC (\%) } & \multicolumn{2}{|l|}{$\Delta \mathrm{FVC}(\%)$} \\
\hline & Control & FGF-2 & Control & FGF-2 & Control & FGF-2 & Control & FGF-2 \\
\hline $\mathrm{T} 1$ & $42.8 \pm 9.7$ & $49.7 \pm 7.4$ & $0.0 \pm 0.0$ & $0.0 \pm 0.0$ & $65.2 \pm 10.9$ & $74.2 \pm 15.2$ & $0.0 \pm 0.0$ & $0.0 \pm 0.0$ \\
\hline $\mathrm{T7}$ & $46.6 \pm 13.6$ & $55.2 \pm 5.3$ & $3.7 \pm 6.6$ & $5.5 \pm 7.8$ & $66.0 \pm 17.7$ & $76.3 \pm 9.9$ & $0.9 \pm 8.3$ & $2.1 \pm 14.7$ \\
\hline $\mathrm{T} 15$ & $42.1 \pm 12.5$ & $52.3 \pm 5.9$ & $-0.7 \pm 7.1$ & $2.5 \pm 8.7$ & $62.4 \pm 15.1$ & $74.5 \pm 11.3$ & $-2.8 \pm 7.5$ & $0.3 \pm 12.9$ \\
\hline A4 & $45.4 \pm 8.3$ & $50.9 \pm 6.8$ & $2.5 \pm 7.2$ & $1.1 \pm 10.1$ & $66.3 \pm 10.3$ & $75.3 \pm 8.5$ & $1.1 \pm 7.9$ & $1.1 \pm 14.6$ \\
\hline
\end{tabular}

FEV ${ }_{1}$ forced expiratory volume in one second, FVC forced vital capacity, FGF-2 fibroblast growth factor-2, T1 1 day of treatment, T7 7 days of treatment, T15 15 days of treatment, $A 4$ after 4 weeks of treatment

a

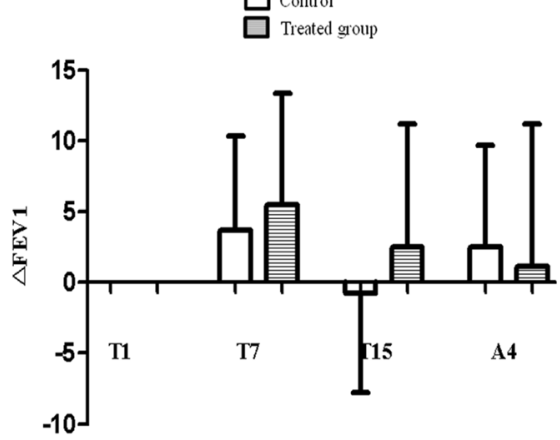

b

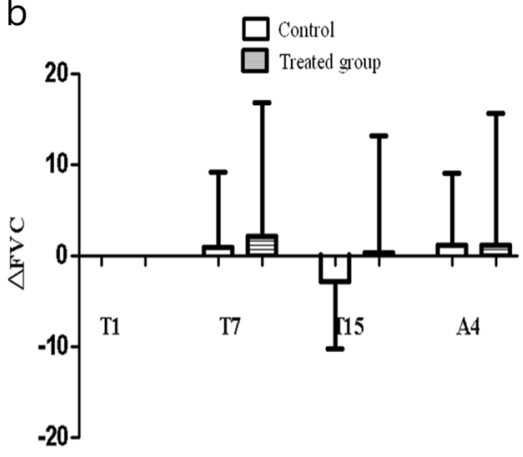

Fig. 8 Change in pulmonary function tests (PFTs) [a change in forced expiratory flow in $1 \mathrm{~s}\left(\triangle \mathrm{FEV} \mathrm{V}_{1}\right.$ ) and $\mathbf{b}$ change in forced vital capacity $(\triangle \mathrm{FVC})$ ] during and after fibroblast growth factor (FGF)-2 treatment. PFTs, including FEV1 and FVC, tended to improve during the recombinant (r) FGF-2 treatment period but not significantly between the rFGF-2 group and the control group $(p>0.05)$

Table 3 ATS scale and $\triangle A T S$ scale of each group during and after FGF-2 treatment in a clinical trial

\begin{tabular}{|c|c|c|c|c|}
\hline & \multicolumn{2}{|c|}{ ATS scale } & \multicolumn{2}{|c|}{$\Delta$ ATS scale } \\
\hline & Control & FGF-2 & Control & FGF-2 \\
\hline $\mathrm{T} 1$ & $2.2 \pm 0.4$ & $2.2 \pm 0.8$ & $0.0 \pm 0.0$ & $0.0 \pm 0.0$ \\
\hline $\mathrm{T7}$ & $1.6 \pm 1.1$ & $2.2 \pm 0.8$ & $-0.6 \pm 0.9$ & $0.0 \pm 0.0$ \\
\hline $\mathrm{T} 15$ & $1.8 \pm 0.8$ & $2.0 \pm 0.0$ & $-0.4 \pm 0.5$ & $-0.2 \pm 0.8$ \\
\hline A4 & $1.4 \pm 0.9$ & $1.5 \pm 0.8$ & $-0.8 \pm 0.4$ & $-0.7 \pm 0.8$ \\
\hline
\end{tabular}

ATS American Thoracic Society, FGF-2 fibroblast growth factor-2, T1 1 day of treatment, $T 77$ days of treatment, $T 1515$ days of treatment, $A 4$ after 4 weeks of treatment.

smoke. Therefore, we believe that a short-term exposure animal model is effective for examining the effects of FGF-2 inhalation.

In this study, BAL fluid demonstrated macrophagedominant inflammation and alveolar destruction of lung tissues according to the MLI. An increase in the number of inflammatory cells, mainly macrophages, was seen in the BAL fluid of smoke-exposed mice. Cigarette smoke is
Table 4 Modified Borg scale and $\Delta$ Modified Borg scale of each group during and after FGF-2 treatment in a clinical trial

\begin{tabular}{llllll}
\hline & \multicolumn{2}{l}{ Modified Borg scale } & & \multicolumn{2}{l}{$\Delta$ Modified Borg scale } \\
\cline { 2 - 3 } \cline { 6 - 6 } & Control & FGF-2 & & Control & FGF-2 \\
\hline T1 & $4.0 \pm 1.2$ & $4.8 \pm 2.0$ & & $0.0 \pm 0.0$ & $0.0 \pm 0.0$ \\
T7 & $3.2 \pm 1.9$ & $4.0 \pm 1.1$ & & $-0.8 \pm 1.3$ & $-0.8 \pm 1.2$ \\
T15 & $2.6 \pm 1.8$ & $3.3 \pm 0.5$ & & $-1.4 \pm 1.1$ & $-1.5 \pm 1.9$ \\
A4 & $2.6 \pm 2.1$ & $2.5 \pm 1.4$ & & $-1.4 \pm 1.1$ & $-2.3 \pm 1.9$ \\
\hline
\end{tabular}

FGF-2 fibroblast growth factor-2, $T 11$ day of treatment, $T 77$ days of treatment, T15 15 days of treatment, A4 after 4 weeks of treatment.

a well-defined common pollutant that influences cellular components in BAL fluid ${ }^{27,28}$.

Smokers have a significantly elevated number of cells in the lower respiratory tract, mainly due to the increased number of alveolar macrophage ${ }^{29,30}$. Macrophages are the first line of defense against inhaled pollutants, including tobacco smoke. The MLI, which is the standard technique for measuring alveolar destruction, was 
significantly increased in smoke-exposed mice compared with normal air-exposed mice.

Intranasal FGF-2 administration had a protective effect on emphysema: (1) a decline in the number of inflammatory cells, especially macrophages and lymphocytes, in BAL fluid; and (2) a decline in alveolar destruction and a regenerative effect in emphysematous mice. These results indicate that loss of FGF-2 production is an important mechanism for developing COPD in response to smoke. Caspase-3/7 activity, which is related to lung cell apoptosis, was significantly decreased in the mice after FGF-2 treatment. Previous studies have shown that FGF-2 prevents apoptosis induced by chemotherapy in patients with lung cancer ${ }^{31,32}$. Intravascular FGF-GMS administration reduces dilatation of the alveolar space and microvascular proliferation in the emphysematous canine lung ${ }^{16}$. Intratracheal administration of FGF-2 increases blood flow in damaged lungs and improves blood gas values, thus allowing recovery of pulmonary function in a canine emphysema model $^{17,18}$. Intrapleural administration of FGF-2 in rats with emphysematous lungs induces lung regeneration ${ }^{19}$. These studies suggest a new potential emphysema treatment using FGF-2, and the present study confirmed these findings. However, the positive effects of FGF-2 in animal studies may not be equally applicable to human COPD. Therefore, we further studied human patients with COPD to determine whether FGF-2 is involved in the pathogenesis of COPD.

In our study, the analysis of FGF-2 in human COPD showed decreased plasma levels of FGF-2 in patients with COPD compared with normal subjects. The mean plasma FGF-2 level was $102.9 \mathrm{pg} / \mathrm{mL}$ in patients with COPD and $125.5 \mathrm{pg} / \mathrm{mL}$ in the healthy controls $(P=0.037)$. When we consider these marginal differences in FGF-2 levels, other factors involved in the development of COPD should be considered. However, when considering the results of animal experiments together, there might be a protective effect of FGF-2, although FGF-2 levels between COPD patients and healthy controls were marginal. Our findings of downregulated FGF-2 indicate that the compensatory mechanisms observed in mice are also active in patients with COPD, as smoking has been suggested to have a strong effect on the imbalance of proteases/antiproteases, including elastases and collagenases, and extracellular matrix deposition in the lungs. FGF-2 in the bronchial epithelium could be involved in the proliferation and repair of epithelial cells after injury in patients with COPD. However, FGF-2 could also be involved in fibrosis and tissue remodeling in patients with asthma and $\mathrm{COPD}^{33}$.

Airway remodeling is an important pathophysiological feature of COPD as well as asthma and is characterized by thickening of the airway wall with increased collagen deposition and airway smooth muscle hypertrophy and hyperplasia $^{34,35}$. The mechanisms underlying airway remodeling are not well understood. One molecule with possible relevance is FGF-2, which is a potent mitogen of fibroblasts, airway smooth muscle cells, and endothelial cells. The degree of airway wall thickening is associated with disease progression, which is the main cause of decreased lung function in patients with COPD because remodeling reduces airflow and distensibility ${ }^{36-38}$. FGF-2 plays an important role in wound healing by promoting the migration and proliferation of fibroblasts, reversing myofibroblast phenotypes, and regenerating airway epithelial cells ${ }^{11,39}$. In particular, FGF-2 has an inhibitory effect on the differentiation of fibroblasts into myofibroblasts, suggesting a possible anti-scarring effect of FGF-2 during wound healing.

Furthermore, we conducted a clinical trial in patients with COPD to determine the effect and safety of inhaled rFGF-2. The results showed that inhaled rFGF-2 was safe and well tolerated in patients with COPD. No significant differences were observed in terms of AEs associated with the inhaled rFGF-2 treatment compared with the placebo during the study period. Only 2 patients developed minimal side effects of cough and sore throat, which were self-limiting.

The present clinical study had several limitations. First, the inclusion of a small number of patients from a single center showed the potential for selection bias. Despite this limitation, this pilot clinical trial is the first one done in humans as a preclinical study. Further large-scale clinical studies are warranted. Second, objective airway inflammatory markers, including inflammatory cells in the sputum, cytokines, and chemokines, were not measured in the clinical trial. However, when the animal experimental results are considered together, rFGF-2 may be effective at suppressing inflammatory changes. Third, there is a paucity of data on the safety, efficacy, and pulmonary pharmacokinetics of rFGF-2 administered via the inhalation route. We were unaware of the concentrations or distribution of rFGF-2 in the pulmonary system. Fourth, our study did not assess long-term safety or patient outcomes, such as the exacerbation rate after completion of treatment. However, clinical improvements and no serious AEs 4 weeks after treatment could be considered reasonable treatment goals for COPD. Finally, we did not evaluate correlations between FGF-2 and lung function/clinical parameters. Large-scale clinical trials should be done in the future.

COPD remains an inextirpable disease with significant morbidity and mortality rates when accompanied by acute exacerbations. Unfortunately, no fundamental treatment agent has been identified. Currently, several drugs targeting the small airways of patients with COPD have been developed and successfully used, but there are no effective drugs for emphysema. The results of this study demonstrate that FGF-2 is involved in the pathogenesis of 
emphysema; therefore, rFGF-2 is a therapeutic option for Korean patients with emphysema-type COPD. The potential attenuation of symptom progression associated with COPD reported in this study makes rFGF-2 a reasonable agent for further prospective trials.

\section{Acknowledgements}

This study was supported by a grant from the Korean Health Technology R\&D Project, Ministry of Health and Welfare, Republic of Korea (HI14C2628).

\section{Author details}

${ }^{1}$ Department of Pulmonary and Critical Care Medicine, Asan Medical Center, University of Ulsan College of Medicine, Seoul, Republic of Korea. ${ }^{2}$ Department of Internal Medicine, Dankook University College of Medicine, Cheonan, Republic of Korea. ${ }^{3}$ Institute of MD Healthcare, Inc, Seoul, Republic of Korea

\section{Conflict of interest}

The authors declare that they have no conflict of interest.

\section{Publisher's note}

Springer Nature remains neutral with regard to jurisdictional claims in published maps and institutional affiliations.

Supplementary information accompanies this paper at https://doi.org/ 10.1038/s12276-018-0178-y.

Received: 4 March 2018 Revised: 22 August 2018 Accepted: 4 September 2018.

Published online: 14 November 2018

\section{References}

1. Global Strategy for the Diagnosis, Management and Prevention of COPD, Global Initiative for Chronic Obstructive Lung Disease (GOLD) 2018. Available at: http://www.goldcopd.org/.

2. Wedzicha, J. A. et al. Indacaterol-glycopyrronium versus salmeterol-fluticasone for COPD. N. Engl. J. Med 374, 2222-2234 (2016).

3. Janson, C. et al. Pneumonia and pneumonia related mortality in patients with COPD treated with fixed combinations of inhaled corticosteroid and long acting $\beta 2$ agonist: observational matched cohort study (PATHOS). BMJ 346, f3306 (2013).

4. Morse, D. \& Rosas, I. O. Tobacco smoke-induced lung fibrosis and emphysema. Annu Rev. Physiol. 76, 493-513 (2014).

5. Talikka, M. et al. Genomic impact of cigarette smoke, with application to three smoking-related diseases. Crit. Rev. Toxicol. 42, 877-889 (2012).

6. MacNee, W. Oxidants/antioxidants and COPD. Chest 117(5Suppl 1) 303S-317SS (2000).

7. Celedón, J. C. et al. The transforming growth factor-beta1 (TGFB1) gene is associated with chronic obstructive pulmonary disease (COPD). Hum. Mol. Genet 13, 1649-1656 (2004).

8. Chen, P. Y. et al. Fibroblast growth factor (FGF) signaling regulates transforming growth factor beta (TGF $\beta$ )-dependent smooth muscle cell phenotype modulation. Sci. Rep. 6, 33407 (2016).

9. Lee, B. J. et al. Protective effects of basic fibroblast growth factor in the development of emphysema induced by interferon- $\gamma$. Exp. Mol. Med. 43, 169-178 (2011).

10. Bikfalvi, A., Klein, S., Pintucci, G. \& Rifkin, D. B. Biological roles of fibroblast growth factor-2. Endocr. Rev. 18, 26-45 (1997).

11. Nugent, M. A. \& lozzo, R. V. Fibroblast growth factor-2. Int. J. Biochem. Cell Biol. 32, 115-120 (2000).

12. Burgess, J. K. The role of the extracellular matrix and specific growth factors in the regulation of inflammation and remodelling in asthma. Pharmacol. Ther. 122, 19-29 (2009).

13. Hoshino, M., Takahashi, M. \& Aoike, N. Expression of vascular endothelial growth factor, basic fibroblast growth factor, and angiogenin immunoreactivity in asthmatic airways and its relationship to angiogenesis. J. Allergy Clin. Immunol. 107, 295-301 (2001).
14. Redington, A. E. et al. Basic fibroblast growth factor in asthma: measurement in bronchoalveolar lavage fluid basally and following allergen challenge. J. Allergy Clin. Immunol. 107, 384-387 (2001).

15. Shute, J. K. et al. Epithelial expression and release of FGF-2 from heparan sulphate binding sites in bronchial tissue in asthma. Thorax 59, 557-562 (2004).

16. Chang, S. S., Yokomise, H., Matsuura, N., Gotoh, M. \& Tabata, Y. Novel therapeutic approach for pulmonary emphysema using gelatin microspheres releasing basic fibroblast growth factor in a canine model. Surg. Today $\mathbf{4 4}$, 1536-1541 (2014).

17. Morino, S. et al. Fibroblast growth factor-2 promotes recovery of pulmonary function in a canine models of elastase-induced emphysema. Exp. Lung Res. 33, 15-26 (2007)

18. Morino, S. et al. Fibroblast growth factor- 2 induces recovery of pulmonary blood flow in canine emphysema models. Chest 128, 920-926 (2005).

19. Kawago, M. et al. Intrapleural administration of gelatin-embedded, sustainedrelease basic fibroblast growth factor for the regeneration of emphysematous lungs in rats. J. Thorac. Cardiovasc. Surg. 147, 1644-1649 (2014).

20. Jeon, S. G. et al. Recombinant basic fibroblast growth factor inhibits the airway hyperresponsiveness, mucus production, and lung inflammation induced by an allergen challenge. J. Allergy Clin. Immunol. 119, 831-837 (2007).

21. Kim, Y. S. et al. The safety and efficacy of recombinant fibroblast growth factor 2 in human asthmatics: a pilot study. Allergy Asthma Respir. Dis. 2, 200-207 (2014).

22. Huh, J. W. et al. Bone marrow cells repair cigarette smoke-induced emphysema in rats. Am. J. Physiol. Lung Cell. Mol. Physiol. 301, L255-L266 (2011).

23. Kim, Y. S. et al. Extracellular vesicles, especially derived from Gram-negative bacteria, in indoor dust induce neutrophilic pulmonary inflammation associated with both Th1 and Th17 cell responses. Clin. Exp. Allergy 43, 443-454 (2013).

24. Lazarous, D. F. et al. Effects of chronic systemic administration of basic fibroblast growth factor on collateral development in the canine heart. Circulation 91, 145-153 (1995).

25. Schaper, W., De Brabander, M. \& Lewi, P. DNA synthesis and mitoses in coronary collateral vessels of the dog. Circ. Res. 28, 671-679 (1971).

26. Partridge, M. R. et al. Development and validation of the capacity of daily living during the morning questionnaire and the Global Chest Symptoms Questionnaire in COPD. Eur. Respir. J. 36, 96-104 (2010).

27. Lommatzsch, M. et al. Acute effects of tobacco smoke on human airway dendritic cells in vivo. Eur. Respir. J. 35, 1130-1136 (2010).

28. Costabel, U. \& Guzman, J. Effect of smoking on bronchoalveolar lavage constituents. Eur. Respir. J. 5, 776-779 (1992).

29. Kuschner, W. G., D'Alessandro, A., Wong, H. \& Blanc, P. D. Dose-dependent cigarette smoking-related inflammatory responses in healthy adults. Eur. Respir. J. 9, 1989-1994 (1996)

30. Burke, W. M. et al. Smoking-induced changes in epithelial lining fluid volume, cell density and protein. Eur. Respir. J. 5, 780-784 (1992).

31. Pardo, O. E. et al. Fibroblast growth factor-2 induces translational regulation of $\mathrm{BCl}-\mathrm{XL}$ and $\mathrm{BCl}-2$ via a MEK-dependent pathway: correlation with resistance to etoposide-induced apoptosis. J. Biol. Chem. 277, 12040-12046 (2002).

32. Pardo, O. E. et al. Fibroblast growth factor 2-mediated translational control of IAPs blocks mitochondrial release of Smac/DIABLO and apoptosis in small cell lung cancer cells. Mol. Cell Biol. 23, 7600-7610 (2003).

33. Aubry, M. C., Wright, J. L. \& Myers, J. L. The pathology of smoking related lung diseases. Clin. Chest Med. 21, 11-35 (2000). vii.

34. Sun, C. et al. LL-37 secreted by epithelium promotes fibroblast collagen production: a potential mechanism of small airway remodeling in chronic obstructive pulmonary disease. Lab. Invest. 94, 991-1002 (2014).

35. Kim, S. H. et al. Perceptions of severe asthma and asthma-COPD overlap syndrome among specialists: a questionnaire survey. Allergy Asthma Immunol. Res. 10, 225-235 (2018).

36. Krimmer, D. I., Burgess, J. K., Wooi, T. K., Black, J. L. \& Oliver, B. G. Matrix proteins from smoke-exposed fibroblasts are pro-proliferative. Am. J. Respir. Cell Mol. Biol. 46, 34-39 (2012).

37. Hogg, J. C. et al. The nature of small-airway obstruction in chronic obstructive pulmonary disease. N. Engl. J. Med. 350, 2645-2653 (2004).

38. James, A. L. \& Wenzel, S. Clinical relevance of airway remodelling in airway diseases. Eur. Respir. J. 30, 134-155 (2007).

39. Turner, N. \& Grose, R. Fibroblast growth factor signalling: from development to cancer. Nat. Rev. Cancer 10, 116-129 (2010). 